\title{
Palm tocotrienol rich fraction with palm kernel oil supplementation prevents development of liver steatosis in high fat diet ICR mice
}

\author{
Mohd Danial Mohd Efendy Goon ${ }^{1,2,3}$, Nur Izzati Zulkanain ${ }^{1,2}$, Siti Hamimah Sheikh Abdul Kadir ${ }^{1,2,3}$, \\ Sharaniza Ab Rahim ${ }^{1}$, Musalmah Mazlan ${ }^{1}$, Normala Abd Latip ${ }^{4}$, Mardiana Abdul Aziz ${ }^{5}$, Norizal Mohd Noor ${ }^{5}$ \\ ${ }^{1}$ Department of Biochemistry, Faculty of Medicine, Universiti Teknologi MARA (UiTM), Cawangan Selangor, Sungai Buloh, Selangor, Malaysia; \\ ${ }^{2}$ Institute for Pathology, Laboratory and Forensics (I-PPerForM), Universiti Teknologi MARA (UiTM), Cawangan Selangor, Sungai Buloh, \\ Selangor, Malaysia; ${ }^{3}$ Institute of Molecular Medicine Biotechnology, Faculty of Medicine, Universiti Teknologi MARA (UiTM), Cawangan Selangor, \\ Sungai Buloh, Selangor, Malaysia; ${ }^{4}$ Atta-ur-Rahman Institute for Natural Products Discovery (AuRIns), Faculty of Pharmacy, Universiti Teknologi \\ MARA (UiTM), Cawangan Selangor, Sungai Buloh, Selangor, Malaysia; ${ }^{5}$ Department of Pathology, Faculty of Medicine, Universiti Teknologi \\ MARA (UiTM), Cawangan Selangor, Sungai Buloh, Selangor, Malaysia \\ Contributions: (I) Conception and design: SH Sheikh Abdul Kadir, S Ab Rahim, M Mazlan, MD Mohd Efendy Goon; (II) Administrative support: \\ MD Mohd Efendy Goon, NI Zulkanain; (III) Provision of study materials or patients: SH Sheikh Abdul Kadir, S Ab Rahim, M Mazlan; (IV) \\ Collection and assembly of data: MD Mohd Efendy Goon, NI Zulkanain; (V) Data analysis and interpretation: All authors; (VI) Manuscript \\ writing: All authors; (VII) Final approval of manuscript: All authors. \\ Correspondence to: Siti Hamimah Sheikh Abdul Kadir. Department of Biochemistry, Faculty of Medicine, Universiti Teknologi MARA (UiTM), \\ Cawangan Selangor, 47000 Sungai Buloh, Selangor, Malaysia. Email: sitih587@salam.uitm.edu.my.
}

Background: The prevalence of non-alcoholic fatty liver disease (NAFLD) in Asian countries is increasing at concerning level. Currently, no specific treatment available to prevent its oxidative stress and progression except for diet and lifestyle changes. Vitamin E such as tocotrienol-rich fraction (TRF) has a promising potential in preventing NAFLD progression. TRF is a potent antioxidant but has low bioavailability due to the use of long-chain triglycerides (LCT) as its carrier. Testing of potential therapeutic agents such as TRF are commonly carried out using animal models. These animal models are often costly due to limited access to the supply especially Asian countries and predisposed to high transportation cost. Lower expenditure of NAFLD model should be investigated without forfeiting the outcome of study. Therefore, this study addresses the gap by utilizing the ICR mice as NAFLD model through dietary modification and testing on the newly formulated TRF with combination of palm kernel oil (PKO) as a medium-chain triglycerides (MCT) carrier.

Methods: Fifteen ICR strain mice were randomly group into two control and one treatment group. Control groups received high-fat diet (HFD) only and standard diet while treatment group was given HFD with TRF (200 mg/kg/day). Study was carried out for 10 weeks. Weights were recorded twice a week. At the end of study, all mice were euthanized and data such weights, waist circumference and random blood glucose were recorded. Liver from each mouse were prepared for histology assessment.

Results: Mice mean weights and random blood sugar showed no difference between group $(\mathrm{P}>0.05)$ while significance waist circumference was larger in HFD and TRF groups compared to $\mathrm{SD}(\mathrm{P}<0.05)$. Histology assessment showed steatosis in TRF group had lower severity compared to HFD group. NAFLD activity score (NAS) was lower in treatment group compared to HFD group.

Conclusions: TRF showed promising potential as an agent to reduce NAFLD progression in ICR mice. Further study at gene and protein levels are required to fully elucidate the mechanism of this new TRF formulation in reducing NAFLD progression.

Keywords: Tocotrienol; anti-oxidant; fatty liver; medium-chain triglycerides; inbred ICR mice

Received: 11 October 2019; Accepted: 11 February 2020; Published: 25 January 2022.

doi: $10.21037 / \operatorname{tgh} .2020 .02 .20$

View this article at: http://dx.doi.org/10.21037/tgh.2020.02.20 


\section{Introduction}

Non-alcoholic fatty liver disease (NAFLD) is characterized by pathologic accumulation of triglycerides in the liver known as hepatic steatosis. Hepatic steatosis in NAFLD occurs without or minimal intake of alcohol, void of genetic syndrome affecting the liver and the individual was not subjected to drug-induced liver injury. NAFLD may remain benign for a period of time but may progress to severe condition such as NASH-related cirrhosis, fibrosis and cancer. NAFLD development is highly seen in individuals with insulin resistance, obesity, essential hypertension, low level of high-density lipoprotein (HDL) and hypertriglyceridemia (1-3). Patients with metabolic syndrome has four to eleven times higher risk in developing NAFLD (4). Recent report showed increasing prevalence of NAFLD in Asian countries. It is estimated that the prevalence of NAFLD recorded at 26.8\% from 2011 to 2015 (5). The mainstay treatment of NAFLD is weight loss, to interrupt further inflammation and necrosis, resulting in fibrosis of the liver. Currently, there are no Food and Drug Administration-approved treatment for NAFLD (6). Vitamin E and pioglitazone are the options available $(7,8)$. However, side effects of this regimen exist such as weight gain secondary to pioglitazone and poor compliance. Vitamin E isoforms such as tocotrienol and tocopherol have shown to reduce oxidative stress, inflammation and injury in liver (9-12). It is established that tocotrienol is superior in terms of its anti-oxidant effect compared to tocopherol $(13,14)$. Tocotrienol-rich fractions (TRF) has been under investigation for its attribute in controlling oxidative stress in tissue but the bioavailability is subject to discussion $(15,16)$. Previously, TRF was delivered by using long-chain triglycerides (LCT) such as palm oil and olive oil $(17,18)$. However, the metabolic pathway taken by LCT require packaging into chylomicrons and transported along the lymphatic system and systemic circulation before reaching the hepatic tract $(19,20)$. Medium-chain triglycerides (MCT), on the other hand, promote simpler route comprises of rapid absorption into the intestinal epithelial cells and transported directly into the portal vein, bypassing the lymphatic and systemic circulation (19). Investigating NAFLD and potential therapeutic agents require the utilization of animal model. Understanding of the induced therapy can be elucidated through the animal's serum, urine, and organ tissues. Nevertheless, studies involving animal model often render to be costly due to limited options available in terms of the animal supply and transportation especially in developing countries. Lower cost of the NAFLD model should be formed without forfeiting the outcome of research. Apart from that, animal model selected for a study should reflect human condition in order to elucidate the safety and adverse effects of tested drugs prior to clinical trial. Therefore, our study presents the use of NAFLD animal model through ICR mice subjecting to high-fat diet and applying the newly formed enhanced formulation of TRF using PKO as its carrier. PKO is an MCT that often disregard as part of drug formulation, originating from the kernel of palm fruit. In combination of this MCT with TRF, the formulation would able to prevent further deterioration of NAFLD.

\section{Methods}

\section{Materials}

\section{Mice and diet}

This study was carried out using 15 male ICR strain mice at six-weeks old (A-Sapphire Sdn Bhd, Malaysia). Prior to study, they were acclimatized for a week. They were provided with unlimited access to standard diet and reverse-osmosis (RO) water during acclimatization period. Mice were handled according to the guideline outlined by the UiTM Animal Care and Use Committee [approved protocol number: UiTM CARE 4/2018/ (251/2018)]. They were placed separately, each in plastic cage with steel top cover. The room accommodating them had temperature controlled at $22 \pm 1{ }^{\circ} \mathrm{C}$ with relative humidity of $60 \% \pm 5 \%$ and strict 12 hours of light and dark cycles daily.

\section{Blood collection and tissue collection}

Blood collection was performed using $1 \mathrm{~mL}$ syringes with 25 G needle (Terumo, Belgium). Vacutainer (red-top tubes, $\mathrm{BD}$, Malaysia) were utilized for blood sample collection. Serum was acquired by centrifuging the vacutainer at $10,000 \mathrm{G}$ for 15 minutes in $4{ }^{\circ} \mathrm{C}$. Serum was transferred and placed into $1.5 \mathrm{~mL}$ reaction tube (Greiner BioOne, Austria). Liver tissue was fixed using $10 \%$ neutral buffered formalin (Leica Biosystem, Germany). Random blood glucose was recorded using Accu-chek Performa 2 glucometer (Roche, Switzerland) with Accu-chek Guide Test Strips (Roche, Switzerland).

\section{TRF}

TRF and stripped palm kernel oil were acquired from SimeDarby Research Sdn. Bhd. (Selangor, Malaysia). The TRF was formulated to contain $80 \%$ tocotrienol and $20 \%$ tocopherol derived from palm fruit. TRF was prepared in a mixture with $\mathrm{PKO}$ at ratio of 1:10. 


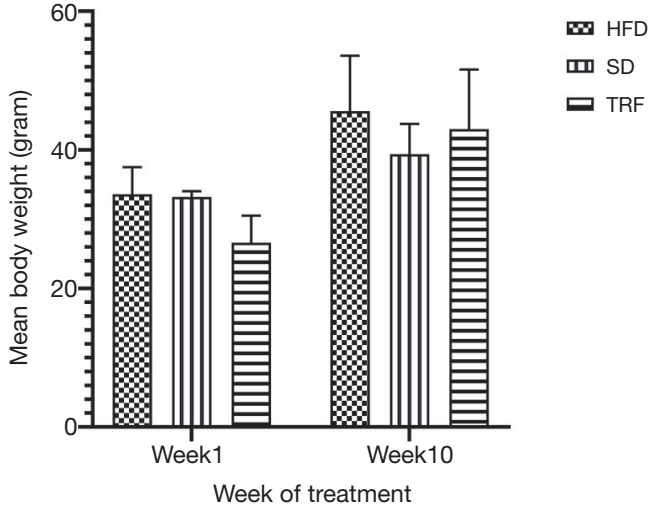

Figure 1 Mice mean body weight during the first and end of study. Mice mean body weights were compared between HFD, SD and TRF group. No difference in mean weight was observed between groups $(\mathrm{P}=0.462)$. HFD, high-fat diet; TRF, tocotrienol-rich fraction; $\mathrm{SD}$, standard diet.

\section{Animal study}

After acclimatization, mice were divided into three equal groups consisting of HFD only group (HFD) (positive control), standard diet (SD) only group (negative control) and HFD with TRF group (TRF) (treatment group). SD group was provided standard diet while HFD and TRF groups were given $60 \%$ high-fat diet along with $\mathrm{RO}$ water ad libitum. As for the TRF group, $200 \mathrm{mg}$ per $\mathrm{kg}$ of TRF, was given by enforcing through a pellet of standard diet once daily. All groups were housed in Laboratory Animal Care Unit (LACU), Institute of Medical Molecular Biotechnology (IMMB), Universiti Teknologi MARA (UiTM), Sg. Buloh Campus, Malaysia. Throughout the 10 -week study, their weight and diet consumption were recorded twice a week. At the end of 10 weeks, all mice were euthanized by rapid cervical dislocation in concordance to the animal ethics guideline. Their blood was drawn by intra-cardiac aspiration using $25 \mathrm{G}$ needle. Serum was acquired and kept for future study. Reading on random blood glucose was acquired by nicking the end of mice's tail to produce drop of blood and recorded using glucometer. Waist circumference was measured and recorded.

\section{Histopatbology assessment}

Liver from each mouse was harvested, sampled from each lobe and stored in 10\% neutral-buffered formalin. The remaining liver and other organs such as kidneys, heart, skin, brain and adipose tissue were harvested, snapped- freeze by liquid nitrogen and stored in $-80{ }^{\circ} \mathrm{C}$ for future study. Liver harvested from each mouse was sample by surgically removed in wedge-shaped on each lobe and fixed with $10 \%$ neutral-buffered formalin for 72 hours. The liver was processed using tissue processor, embedded in paraffin-wax (Surgipath Paraplast Plus, Leica Biosystems, Germany), trimmed and sectioned before being stained with Hematoxylin and Eosin (Leica Biosystems, Germany). Findings from the histopathological assessment were validated by pathologist from Sg. Buloh Hospital, Selangor, Malaysia by single-blinded method.

\section{Statistical analysis}

Statistical analysis of results was performed using SPSS version 25 (IBM, USA). Results are presented as mean \pm SD. Kruskal-Wallis test was used to compare variables between groups. Statistical significance was considered as the $\mathrm{P}$ value of less than $0.05(\mathrm{P}<0.05)$.

\section{Results}

\section{Anthropometric data}

Anthropometric data consist of weight and waist circumference were recorded during the 10 weeks and at end of study, respectively. No difference in mean weight was observed between SD, HFD and TRF groups $(\mathrm{P}=0.462)$. Graph of mean weight of all groups is shown as in Figure 1. Waist circumference of all groups were recorded between 8 and $12 \mathrm{~cm}$. Waist circumference of mice were larger in the HFD and TRF groups compared to mice on SD $(\mathrm{P}=0.019)$. The bar chart of waist circumference of the three groups is illustrated as in Figure 2.

\section{Random blood glucose}

Random blood glucose level was taken during mice euthanasia from tail blood. The random blood glucose recorded highest in TRF group followed by HFD group and SD $(\mathrm{P}=0.075)$. Bar-chart of the random blood glucose of the groups is shown in Figure 3.

\section{Histopathological assessment}

Upon inspection, liver in HFD and TRF groups appeared to be enlarged with substantial deposition of adipose tissue around the abdominal region compared to SD. The liver from HFD group appeared as yellowish-discoloration. 


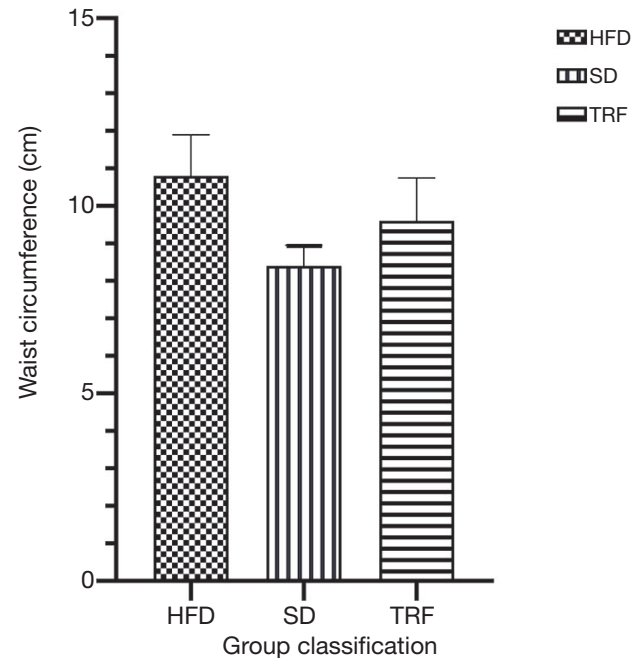

Figure 2 Mice waist circumference measured at the end of study and compared between groups. Waist circumference of mice were larger in the HFD and TRF groups compared to mice on SD $(\mathrm{P}=0.019)$. HFD, high-fat diet; TRF, tocotrienol-rich fraction; SD, standard diet.

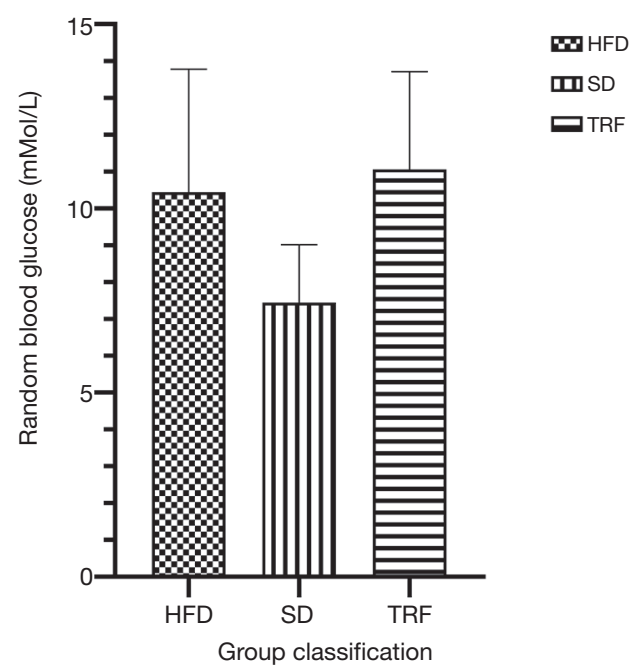

Figure 3 Mice random blood glucose were measured during euthanization using tail blood and readings were compared between groups. The random blood glucose recorded highest in TRF group followed by HFD group and SD ( $\mathrm{P}=0.075)$. HFD, high-fat diet; TRF, tocotrienol-rich fraction; SD, standard diet.

The gross appearance of liver comparison between all groups is shown in Figure 4 to illustrate enlargement of liver (hepatomegaly) and yellow-discoloration compared to normal liver. The liver section from all groups were stained with hematoxylin and eosin. The liver from each mouse was sample in every lobe for histopathology assessment. Assessment and validations of the histology slides showed prominent differences between groups. Assessment of liver tissue in HFD group showed presence of both microvesicular and macrovesicular steatosis. The steatosis appeared diffused encompassing zone one and two. However, higher volume of steatosis concentrated on portal triad than central vein. Histology assessment of liver from TRF group showed evidence of mild to absence of steatosis. The steatosis appeared focally around zone three rather than diffuse. There were more microvesicular steatosis compared to macrovesicular steatosis. To some extent, some of the liver tissue sampled showed no significant histopathological findings with normal hepatocytes morphology and preserved portal triads and central veins similar to the liver morphology presented in SD group. Figure 5 showed the histologic findings described. Scoring based on NAFLD Activity Score (NAS) by NASH Clinical Research Network (CRN) was applied on each histology slides (21). The score is shown in Table 1. Scores from TRF group are generally lower compared to HFD group contributed by lower score in steatosis of parenchymal and lacking in hepatocyte ballooning and lobular inflammation.

\section{Discussion}

This study focused on presenting a preclinical NAFLD model using the ICR mice with $60 \%$ high-fat diet and the effect of TRF with PKO as carrier on the liver. ICR is a mice model that is cheaper compared to other mice used as NAFLD model, especially compared to genetically modified mice. Previously, C57BL/6 mice were preferred as they have the predilection of developing metabolic syndrome and NAFLD induced through dietary modification or chemically (22). NAFLD model using ICR mice showed no shortcomings in developing NAFLD as the fatty liver observed through histology findings showed that the NAFLD developed without progressing rapidly into fibrosis and cirrhosis stage in the allocated time of ten weeks which reflect one of the risk factors in developing NAFLD in human population (3). NAFLD model using ICR mice has been utilized in other study as well, suggesting similar findings in terms of the NAFLD progression (23). Both histological findings in this study and study carried out by Jian et al. (23) fulfill the histopathological features in making the diagnosis of NAFLD (24). Through dietary modification, the development and progression 

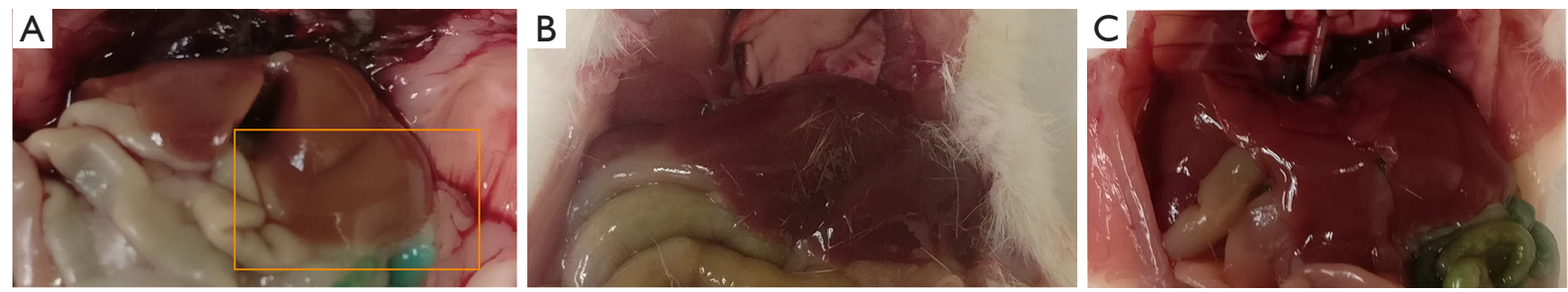

Figure 4 Comparison of mouse liver between HFD group (A) and TRF group (B) and SD group (C). The liver from HFD group showed yellow discoloration (yellow box) on gross examination while the liver from treatment group appeared red as seen in normal mouse liver (C). Both livers appeared enlarged suggesting hepatomegaly compared to normal mouse liver. HFD, high-fat diet; TRF, tocotrienol-rich fraction; SD, standard diet.

$10 \times$
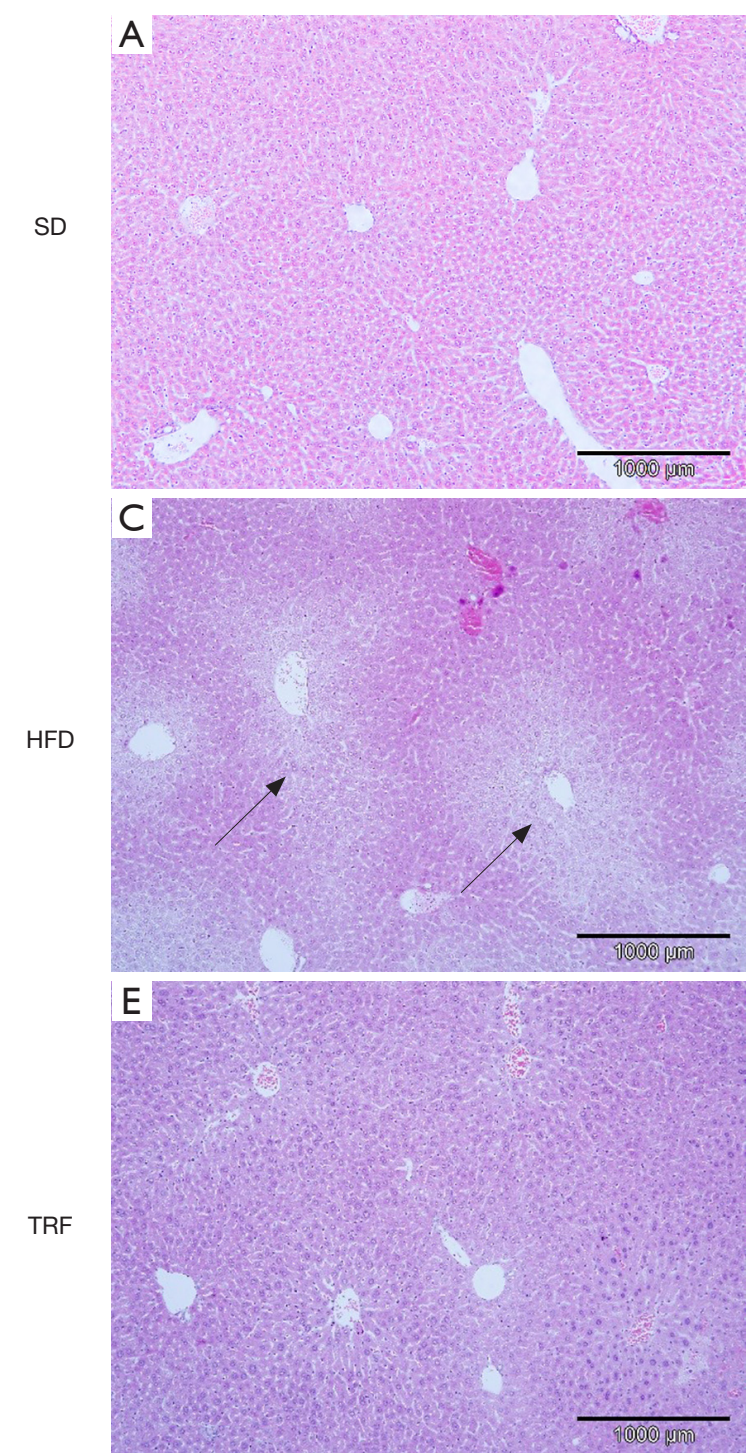

$40 x$
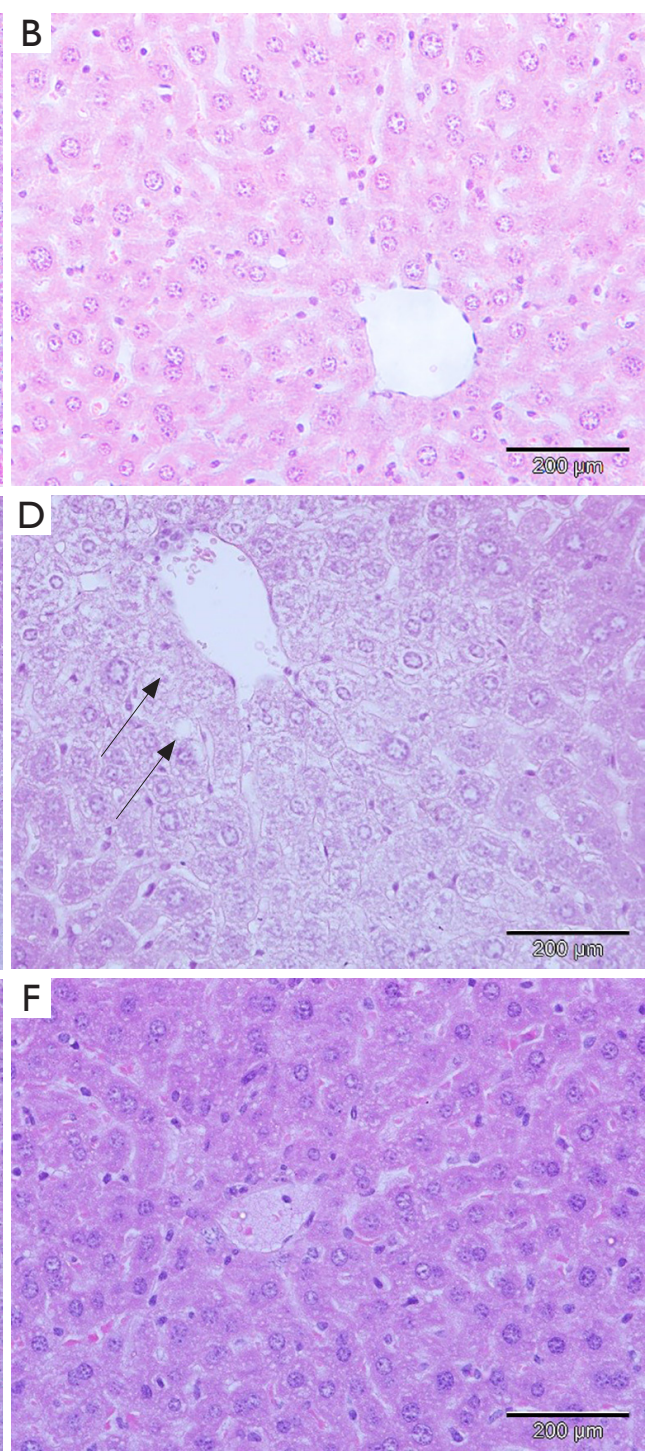

Figure 5 Representative histology of liver from SD (A,B), HFD (C,D) and TRF (E,F) treated group, at 10x and 40x magnification; HE staining. (C,D) Presence of microvesicular and macrovesicular steatosis involving all zones (black arrow) were observed in HFD. Hepatocytes morphology preserved and presence of steatosis was not found in TRF treated group $(\mathrm{n}=5)$. HFD, high-fat diet; TRF, tocotrienol-rich fraction. 
Table 1 Scoring on all livers ( $\mathrm{n}=15)$ using NAFLD Activity Score (NAS)

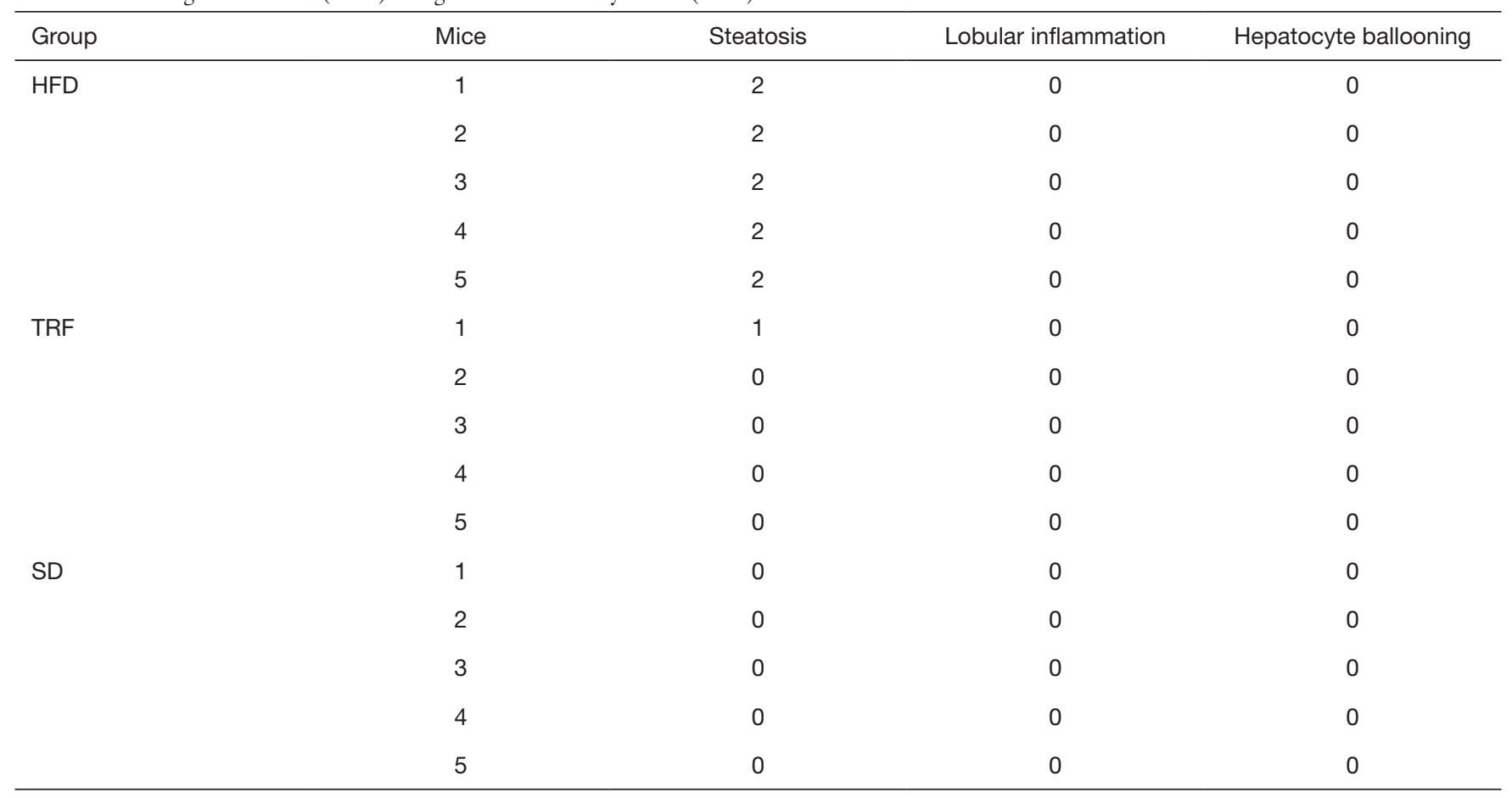

Steatosis is scored based on parenchymal involvement, $0=<5 \%, 1=5-33 \%, 2=34-66 \%, 3=>66 \%$; Lobular inflammation is based on overall assessment of all inflammatory foci, $0=$ no foci, $1=<2$ foci, $2=2-4$ foci, $3=>4$ foci; Hepatocyte ballooning is assessed based on number of cells, $0=$ no ballooning, $1=$ few ballooning cells, $2=$ prominent ballooning. NAFLD, non-alcoholic fatty liver disease; HFD, high-fat diet treated mice group; TRF, Tocotrienol-rich fraction with high-fat diet treated mice group; SD, Mice fed with standard diet only.

of NAFLD in these mice allow adequate time frame for the given treatment to effect. This is important as rapidly developed NAFLD and progression into advanced stage such as NASH and cirrhosis usually exhibited in other types of NAFLD model may prohibit the drug of interest to fully elucidate their therapeutic effect. However, the NAFLD developed in ICR would not be arrested in benign stage as addition in weeks of study, subjected to chronic HFD intake, would develop advanced stages of NAFLD as exhibited in a study by Dowman et al. (25). The treatment in this study, using isoforms of Vitamin E which were tocotrienol and tocopherol, introduced in combination of MCT acting as an agent in preventing NAFLD progressing towards later stages. From the anthropometric data acquired, it can be postulated that TRF does not contribute to weight loss or acts as glucosecontrolling agent. Similar findings have been reported in other studies seen from the use of TRF on animal model $(14,26)$. However, feeding HFD for long period in ICR strain mice have caused them to develop central obesity as the normal waist circumference of mice from the same strain should be less than nine centimeter (27). Central obesity attributed to the risk of developing metabolic syndrome, which has the propensity to develop NAFLD as well (28). In condition of diabetes type 2, Vitamin E exhibited their therapeutic effect by controlling oxidative stress in nerves and tissues rather than lowering serum glucose level (29). Therefore, TRF would be beneficial in diabetes type 2 in controlling neuropathy and nephropathy which both are debilitating on individuals with uncontrolled diabetes type 2 rather than acting as agent in controlling blood glucose level. The full potential of TRF on NAFLD can be exerted as the pathogenesis consist of oxidative stress secondary to lipid peroxidation (30). With multiple evidences that TRF is able to act as lipidlowering agent, combining with anti-oxidant properties, TRF would able to prevent or pacify the progression of NAFLD towards inflammatory process and lipid peroxidation resulting to NASH and fibrosis $(31,32)$. The ability of TRF to prevent NAFLD progression is likely to be contributed by incorporation of MCT allowing quicker delivery into the liver through efficient absorption 
providing higher bioavailability of TRF in exhibiting its anti-oxidant activities $(33,19)$. On top of that, higher percentage of tocotrienol in TRF would be among the factor contributed to effective prevention of NAFLD progression. Tocotrienol was found to be transported in fatty acid rich fractions containing chylomicrons and HDL cholesterol after subjected to TRF in a study. Compared to tocopherol, tocotrienol was detected higher in HDL cholesterol before clearance as well as easily distributed in organs and tissues high in adipose content especially in fatty liver $(15,16,34)$. This is also evident from the presentation of liver histology in the treatment group compared to control group as the NAS score is lower. The reason of lower score in TRF treated group compared to control group would primarily contribute by the high bioavailability and effective anti-oxidant properties delivered by TRF with the help of MCT. Other study has shown this ability through the use of TRF, however, due to the formulation involving LCT, the full potential of its effect was not truly unveiled, contrast to our study (10). By using NAS to score the degree of severity of NAFLD, it provided insights to aminotransferase levels as NAS score was found to correlate with aminotransferase (21). Higher score of NAS suggests higher risk of NAFLD to progress to NASH and cirrhosis. The limitation in this study is the use of minimal sample size $(\mathrm{n}=15)$ to deliver the power for statistical significance. Therefore, we will be using 24 mice for the full-scale study in near future, arming each group with eight mice to overcome this limitation. In addition, the knowledge acquired throughout this study in terms of animal handling and care especially the selection of caging system and method of HFD and TRF delivery, allow us to be more prepared and competent in handling the B6Cg.LepOb/J strain mice which will be used in future study. Future directives of this study will include anti-oxidant assays consisting of determining the level of Superoxide Dismutase (SoD) and glutathione and metabolomics profiling of liver from each mouse to determine the pertinent metabolites involved as anti-oxidant in TRF as well as pathways regulated it in preventing deterioration of NAFLD.

\section{Conclusions}

In conclusion, this study demonstrated that ICR mice are suitable option as NAFLD model through dietary modification. The anthropometric and histological findings supported the diagnosis of NAFLD. Apart from that, TRF has shown to be an effective agent in preventing further progression of NAFLD and to some extent, preserving the liver's morphology in mice receiving it. However, TRF was found ineffective in controlling blood glucose level and reducing weight in this animal study. The NAS score showed lower score in treatment group compared to control group suggesting lower risk for NAFLD to progress to more severe stages. Further investigation should be carried out using NAFLD model to elucidate the mechanism of TRF.

\section{Acknowledgments}

Funding: This study was funded by the MITRA Perdana Grant [600-IRMI/PERDANA 5/3/MITRA (007-2018)-2] and Research Initiative Grant (GIP) [600-IRMI/5/3/GIP (053/2018)] through Universiti Teknologi MARA (UiTM, Malaysia) from the Malaysian government (Ministry of Higher Education).

\section{Footnote}

Conflicts of Interest: All authors have completed the ICMJE uniform disclosure form (available at https://tgh.amegroups. com/article/view/10.21037/tgh.2020.02.20/coif). The authors have no conflicts of interest to declare.

Ethical Statement: The authors are accountable for all aspects of the work in ensuring that questions related to the accuracy or integrity of any part of the work are appropriately investigated and resolved. Mice were handled according to the guideline outlined by the UiTM Animal Care and Use Committee [approved protocol number: UiTM CARE 4/2018/ (251/2018)].

Open Access Statement: This is an Open Access article distributed in accordance with the Creative Commons Attribution-NonCommercial-NoDerivs 4.0 International License (CC BY-NC-ND 4.0), which permits the noncommercial replication and distribution of the article with the strict proviso that no changes or edits are made and the original work is properly cited (including links to both the formal publication through the relevant DOI and the license). See: https://creativecommons.org/licenses/by-nc-nd/4.0/.

\section{References}

1. Adams LA, Waters OR, Knuiman MW, et al. NAFLD as a risk factor for the development of diabetes and the metabolic syndrome: an eleven-year follow-up study. Am J 
Gastroenterol 2009;104:861-7.

2. Ryoo JH, Suh YJ, Shin HC, et al. Clinical association between non-alcoholic fatty liver disease and the development of hypertension. J Gastroenterol Hepatol 2014;29:1926-31.

3. Chang Y, Jung HS, Cho J, et al. Metabolically Healthy Obesity and the Development of Nonalcoholic Fatty Liver Disease. Am J Gastroenterol 2016;111:1133-40.

4. Lee SW, Lee TY, Yang SS, et al. The association of nonalcoholic fatty liver disease and metabolic syndrome in a Chinese population. Hepatobiliary Pancreat Dis Int 2017;16:176-80.

5. Younossi ZM, Koenig AB, Abdelatif D, et al. Global epidemiology of nonalcoholic fatty liver disease-Metaanalytic assessment of prevalence, incidence, and outcomes. Hepatology 2016;64:73-84.

6. Hung CK, Bodenheimer HC. Clinics in Liver Disease. Clinics in Liver Disease 2018;22:175-87.

7. Sanyal AJ, Chalasani N, Kowdley KV, et al. Pioglitazone, vitamin $\mathrm{E}$, or placebo for nonalcoholic steatohepatitis. $\mathrm{N}$ Engl J Med 2010;362:1675-85.

8. Morante M, Sandoval J, Gómez-Cabrera MC, et al. Vitamin E deficiency induces liver nuclear factor- B DNAbinding activity and changes in related genes. Free Radic Res 2005;39:1127-38.

9. Phung N, Pera N, Farrell G, et al. Pro-oxidant-mediated hepatic fibrosis and effects of antioxidant intervention in murine dietary steatohepatitis. Int J Mol Med 2009;24:171-80.

10. Kamat JP, Sarma HD, Devasagayam TP, et al. Tocotrienols from palm oil as effective inhibitors of protein oxidation and lipid peroxidation in rat liver microsomes. Mol Cell Biochem 1997;170:131-7.

11. Magosso E, Ansari MA, Gopalan Y, et al. Tocotrienols for normalisation of hepatic echogenic response in nonalcoholic fatty liver: a randomised placebo-controlled clinical trial. Nutr J 2013;12:166.

12. Tan CY, Saw TY, Fong CW, et al. Comparative hepatoprotective effects of tocotrienol analogs against drug-induced liver injury. Redox Biol 2015;4:308-20.

13. Goon JA, Nor Azman NHE, Abdul Ghani SM, et al. Comparing palm oil tocotrienol rich fraction with alphatocopherol supplementation on oxidative stress in healthy older adults. Clin Nutr ESPEN 2017;21:1-12.

14. Matough FA, Budin SB, Hamid ZA, et al. Tocotrienolrich fraction from palm oil prevents oxidative damage in diabetic rats. Sultan Qaboos Univ Med J 2014;14:e95-e103.

15. Fairus S, Nor RM, Cheng HM, et al. Alpha-tocotrienol is the most abundant tocotrienol isomer circulated in plasma and lipoproteins after postprandial tocotrienol-rich vitamin E supplementation. Nutr J 2012;11:5.

16. Fairus S, Nor RM, Cheng HM, et al. Postprandial metabolic fate of tocotrienol-rich vitamin $\mathrm{E}$ differs significantly from that of alpha-tocopherol. Am J Clin Nutr 2006;84:835-42.

17. Yap WN. Tocotrienol-rich fraction attenuates UVinduced inflammaging: A bench to bedside study. J Cosmet Dermatol 2018;17:555-65.

18. Aliahmat NS, Noor MR, Yusof WJ, et al. Antioxidant enzyme activity and malondialdehyde levels can be modulated by Piper betle, tocotrienol rich fraction and Chlorella vulgaris in aging C57BL/6 mice. Clinics (Sao Paulo) 2012;67:1447-54.

19. Caliph SM, Charman WN, Porter CJ. Effect of short-, medium-, and long-chain fatty acid-based vehicles on the absolute oral bioavailability and intestinal lymphatic transport of halofantrine and assessment of mass balance in lymph-cannulated and non-cannulated rats. J Pharm Sci 2000;89:1073-84.

20. von Bonsdorff-Nikander A, Christiansen L, Huikko L, et al. A comparison of the effect of medium- vs. longchain triglycerides on the in vitro solubilization of cholesterol and/or phytosterol into mixed micelles. Lipids 2005;40:181-90.

21. Kleiner DE, Brunt EM, Van Natta M, et al. Design and validation of a histological scoring system for nonalcoholic fatty liver disease. Hepatology 2005;41:1313-21.

22. Takahashi Y, Soejima Y, Fukusato T. Animal models of nonalcoholic fatty liver disease/nonalcoholic steatohepatitis. World J Gastroenterol 2012;18:2300-8.

23. Jian T, Ao X, Wu Y, et al. Total sesquiterpene glycosides from Loquat (Eriobotrya japonica) leaf alleviate highfat diet induced non-alcoholic fatty liver disease through cytochrome P450 2E1 inhibition. Biomed Pharmacother 2017;91:229-37.

24. Takahashi Y, Fukusato T. Histopathology of nonalcoholic fatty liver disease/nonalcoholic steatohepatitis. World J Gastroenterol 2014;20:15539-48.

25. Dowman JK, Hopkins LJ, Reynolds GM, et al. Development of hepatocellular carcinoma in a murine model of nonalcoholic steatohepatitis induced by use of a high-fat/fructose diet and sedentary lifestyle. Am J Pathol 2014;184:1550-61.

26. Allen L, Ramalingam L, Menikdiwela K, et al. Effects of delta-tocotrienol on obesity-related adipocyte hypertrophy, inflammation and hepatic steatosis in high-fat-fed mice. J 
Nutr Biochem 2017;48:128-37.

27. Shen JZ, Ma LN, Han Y, et al. Pentamethylquercetin generates beneficial effects in monosodium glutamateinduced obese mice and C2C12 myotubes by activating AMP-activated protein kinase. Diabetologia 2012;55:1836-46.

28. Salmanroghani H, Salmanroghani R, Nourian M, et al. Evaluation of neck circumference as an easy and reliable predictor for non-alcoholic fatty liver disease. Turk J Gastroenterol 2019;30:163-70.

29. Vitamin E in Neuroprotection Study (VENUS) Investigators, Hor CP, Fung WY, et al. Efficacy of Oral Mixed Tocotrienols in Diabetic Peripheral Neuropathy: A Randomized Clinical Trial. JAMA Neurol 2018;75:444-52. 30. Brunt EM. Pathology of nonalcoholic fatty liver disease.

doi: $10.21037 / \operatorname{tgh} .2020 .02 .20$

Cite this article as: Mohd Efendy Goon MD, Zulkanain NI, Sheikh Abdul Kadir SH, Ab Rahim S, Mazlan M, Abd Latip N, Abdul Aziz M, Mohd Noor N. Palm tocotrienol rich fraction with palm kernel oil supplementation prevents development of liver steatosis in high fat diet ICR mice. Transl Gastroenterol Hepatol 2022;7:2.
Nat Rev Gastroenterol Hepatol 2010;7:195-203.

31. Fu JY, Che HL, Tan DM, et al. Bioavailability of tocotrienols: evidence in human studies. Nutr Metab (Lond) 2014;11:5.

32. Simeone JC, Bae JP, Hoogwerf BJ, et al. Clinical course of nonalcoholic fatty liver disease: an assessment of severity, progression, and outcomes. Clin Epidemiol 2017;9:679-88.

33. Greenberger NJ, Rodgers JB, Isselbacher KJ. Absorption of medium and long chain triglycerides: factors influencing their hydrolysis and transport. J Clin Invest 1966;45:217-27.

34. Gee PT. Unleashing the untold and misunderstood observations on vitamin E. Genes Nutr 2011;6:5-16. 